\title{
Applying vasculitis activity and damage scores in a group of patients with Behçet's disease - clinical utility
}

\author{
Casandra Buzatu, Sanziana Daia-Iliescu, Ioana Saulescu, \\ Ruxandra Ionescu, Daniela Opris-Belinski
}

Department of Internal medicine and Rheumatology, "Sf. Maria" Clinical Hospital,

"Carol Davila" University of Medicine and Pharmacy, Bucharest, Romania

\begin{abstract}
Background. Behçet's disease is a rare type of vasculitis. Validated activity and damage scores were developed for vasculitis patients in order to allow a better way to evaluate disease activity and decide treatment plans. Objective. The main objective was to compare two vasculitis activity scores applied to a group of patients diagnosed with Behcet's disease and establish correlations between them, damage and the need for immunosuppressive therapy. The secondary objective was to evaluate the connection between damage progression, classical immunosuppressant therapy and long-term cortisone use.

Methods. A study was performed on a cohort of patients diagnosed with Behçet's disease according The International Criteria for Behçet's Disease (ICBD) under surveillance in one Rheumatology Centre, from a non-endemic area. Vasculitis activity and damage scores were calculated for each patient.

Results. 20 patients were included in the study, with a mean age of 35.7 years \pm 10.5 years standard deviation(SD), $14(70 \%)$ under the age of 40 , with a male predominance $60 \%$ (12 patients). All patients presented active disease at the time of the diagnosis. Spearman's rank correlation coefficient between BVAS v3 and BDCAF was strong $r=0.862$ and statistically significant $p<0.001$. The outcome analysis after remission was calculated and rank correlation coefficient between VDI, and both BVASv3 and BDCAF was moderate (VDI-BVASv3 $r=0.747, p<$ $0.001, \mathrm{VDI}-\mathrm{BDCAF} r=0.795, \mathrm{p}<0.001)$. As for immunosuppression induction decision and activity scores, the correlation coefficient was moderate ( $r=0.734$ for BVASv3, $r=0.647$ for BDCAF) with $p<0.001$. There was a moderate correlation between immunosuppressive treatment and VDI $(r=0.700, p<0.001)$. The cause of damage (i.e. vasculitis vs. treatment) is not taken into consideration when we calculate VDI. Data analysis showed the presence of mild correlation and no statistical impact between cyclophosphamide treatment duration and damage calculated as VDI $(r=0.474, p=0.36)$. In contrast, when rank correlation coefficient between cortisone therapy and VDI was calculated, a moderate statistical impact was observed $(r=0.609, p<0.001)$.

Conclusions. An objective assessment of disease's activity can be obtained using disease activity indexes. A moderate to strong correlation was obtained between activity indexes, immunosuppressive treatment initiation and damage progression. Comparing the two activity indexes, it resulted that: BVASv3 correlates stronger with the need for immunosuppressive treatment and both of them are equally able to anticipate damage. Damage progression was correlated stronger with long-term cortisone use, rather than immunosuppressive therapy.
\end{abstract}

Keywords: Behçet disease, vasculitis, activity score, damage, immunosuppression

\section{INTRODUCTION}

Behçet's disease is a rare type of vasculitis that involves both arterial and venous blood vessels. It was first described in 1994 as a syndrome characterized by the triad: oral and genital ulcers and ocular inflammation.

Numerous classification criteria were developed and have been published over the years in order to raise awareness in the area of systemic vasculitis and rare diseases. The most popular criteria are those developed by the collaboration of 7 countries in 1990:
International Study Group Criteria for Behçet's Disease (ISG). They have a specificity of $97 \%$ and a sensitivity of $92 \%$. In 2006, an International Team for revision of the International Criteria for BD (27 countries) proposed a new set of criteria that added vascular and neurological lesion and considered that oral ulcers are no more mandatory. This decision improved sensitivity and maintained a fairly good specificity in comparison to the old criteria $(1,2,3)$.

In order to better assess disease activity and damage validated disease activity scores and damage 
measurements were developed over time as a better way to evaluate patients and decide treatment plans. They were applied especially in clinical trials for randomizing patients and for a more objective evaluation. There are scores designed for a great variety of vasculitis like Birmingham Vasculitis Activity Score (BVAS) and others that are more specific like Behçet's Disease Current Activity Form 2006 (BDCAF).

Birmingham vasculitis activity index (BVAS) is a tool largely used, especially in clinical trials. It includes most of the features that can appear in a vasculitis. The new version, BVASv3, is structured in 56 items divided into organ systems. The scoring sheet records the presence or absence of each item and the total score is an indication of the current activity (4).

In particular, for Behçet's disease the most popular activity form is the Behçet's current activity form 2006 (BDCAF2006). The scoring is based on the history of new clinical features present over the previous 4 weeks. With the collaboration of physicians and ophthalmologists with experience in this field, questions were standardized for all parts of the form. The BDCAF2006 scores for oral and genital ulcerations, skin, joint, gastrointestinal lesions, fatigue, headache and type of large-vessel and central nervous system involvement $(5,6)$.

\section{OBJECTIVES}

The main objective was to compare two vasculitis activity scores applied to a group of patients diagnosed with Behçet's disease - a universal score for systemic vasculitis BVASv3 and a Behçet specific score: BDCAF2006- and establish correlations between them, damage calculated as VDI and the need for immunosuppressive therapy.

The secondary objective was to evaluate the connection between damage progression, classical immunosuppressant therapy and long-term cortisone use.

\section{METHODS}

This is a retrospective analysis of observational data recorded from a cohort of patients diagnosed with Behçet's disease admitted in The Department of Internal medicine and Rheumatology, Sfanta Maria Clinical Hospital, Bucharest, Romania.

The ISG1990 and ICBD classification criteria guidelines were used for diagnosis. Patients with BD who visited our Internal medicine and Rheumatology clinic were enrolled in this study. The selection phase identified 20 patients.

The study protocol was approved by the local Ethical Committee.

\section{VARIABLES}

All medical records from the Internal medicine and Rheumatology department from patients diagnosed with Behçet's disease were analyzed.

For the study group, baseline variables were considered and recorded for every patient: demographic data (gender, age etc.), clinical characteristics (oral and genital aphtous lesions, skin lesions, ocular involvement, neurological manifestations, thromboembolic events), genetic testing, therapy data. Complete data were recorded in all patients except for the HLAB51 typing. Pathergy test was performed in all patients that were enrolled.

Data extracted from de medical record showed that the clinical evaluation of all patients has been done in collaboration with a team of specialists in dermatology, ophthalmology, cardiology, neurology.

To evaluate disease activity, all patients data was examined from their first presentation in clinic to follow-up and disease activity indexes (BVASv3 and BDCAF2006) were calculated.

BVAS records only items which have appeared or have worsen during the previous 3 months. A new version of BVAS (v3) consisting of 56 items, divided into 9 systems has been used. On the scoring sheet, data about the presence or absence of each item has been recorded and the maximum score has been calculated. It comprises the following system involvement: cutaneous, mucous/membranes, eyes, ear-nose-throat, cardiovascular, respiratory, gastrointestinal, renal, nervous system, and general symptoms $(4,7)$.

BDCAF2006 is presented as a guideline for the clinical interview being structured in groups of questions. It evaluates different manifestations of active disease 4 weeks prior to assessment (headache, mouth ulcers, genital ulcers, skin lesions, joint involvement and gastrointestinal symptoms; eye involvement; nervous system involvement; major vessel involvement). Only the signs and symptoms that the clinician believes are due to disease activity are scored. A score out of 12 is obtained - the patient's Behçet's disease activity index score - and then is transformed on interval scale. The obtained score 
shows the impairments relating to disease activity $(5,6,8)$.

Damage progression was recorded as vasculitis damage index (VDI) and was calculated for each patient once remission was obtained. The data analysis included clinical, paraclinical and imaging studies, as were ordered by the patient personal physician.

Vasculitis damage index (VDI) is a tool used to record organ damage that has occurred in patients since onset of vasculitis. Attention must be paid to the fact that patients might have previous comorbidities, which must not be scored. When there is a new patient, the score usually is zero, unless they have more than 3 moths of active disease and damage already appeared or became worse since the onset $(7,9,10)$.

\section{STATISTICAL CONSIDERATIONS AND ANALYSIS}

For the statistical analysis we used SPSS 20.0 and Windows Excel. A p value $<0.01$ was conventionally considered significant and numeric variables were described as mean $+/$ - standard deviation or as median. Spearman's correlation coefficients were calculated between the variables (BVAS v3 Score, BDCAF, VDI and immunosuppressive treatment).

\section{RESULTS}

\section{Patients characteristics}

The study group included 20 patients, with ages at the time of the diagnosis between 13 and 60 years and a mean age of 35.7 years \pm 10.5 years standard deviation (SD). 14 patients $(70 \%)$ were under the age of 40 and a male predominance $60 \%$ (12 patients) was observed. Clinical classification criteria were met at the time of diagnosis in only 10 cases (50\%), 8 male and 2 female patients. The pathergy test was performed for all 20 patients and was positive in 13 cases. The genetic marker, HLA B51, was tested in 6 cases and in was positive in 5 patients $(87 \%)$.

TABLE 1. Patient demographic and clinical characteristics $(n=20)$

\begin{tabular}{|l|c|}
\hline Variable & Value \\
\hline Age, yr & 35.5 \\
\hline Sex (male:female) & $12: 8$ \\
\hline \multicolumn{2}{|c|}{ Clinical symptom } \\
\hline Oral ulcer & $20(100)$ \\
\hline Genital ulcer & $13(65.0)$ \\
\hline Skin lesion & $15(75.0)$ \\
\hline \multicolumn{2}{|c|}{ Organ involvement } \\
\hline Eye & $6(30.0)$ \\
\hline Vascular & $6(30.0)$ \\
\hline Joint & $13(65.0)$ \\
\hline Central nervous system & $3(15)$ \\
\hline Pathergy test positivity & $\mathbf{1 3 / 2 0 ( 6 5 . 0 )}$ \\
\hline HLA-B51 positivity & $\mathbf{5 / 6 ( 2 5 )}$
\end{tabular}

\section{Clinical manifestations}

The presence of mucocutaneous manifestations, in particular recurrent oral aphthous lesions, are one of the most characteristic clinical manifestation of Behçet's disease, one of the items that even makes the physician consider this vasculitis for the differential diagnosis. In the study group all patients presented oral ulcerations $(100 \%)$. As for genital ulcerations, they were present in 13 cases $(65 \%)$. Other skin lesions were identified also: 7 patients $(35 \%)$ presented erythema nodosum, 8 (40\%) pseudofolliculitis.

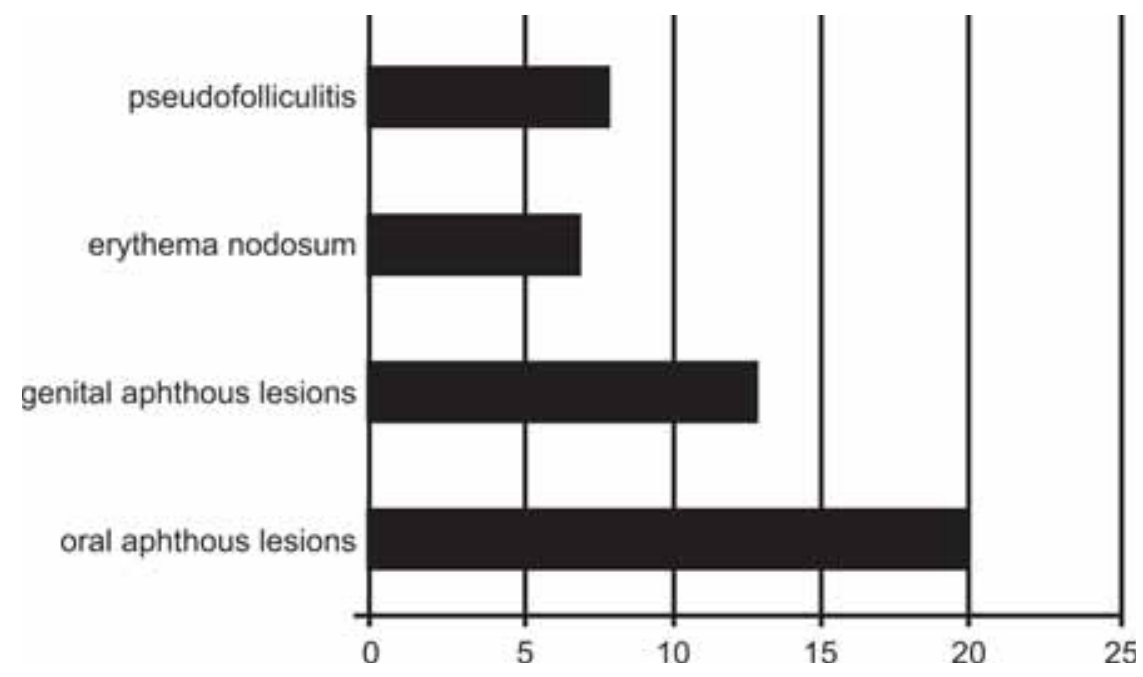

GRAPHIC 1. Mucocutaneous manifestations 


\section{Musculoskeletal involvement}

Some of the most common rheumatic manifestations of Behçet's disease are the presence of arthralgia and arthritis with prevalence that varies, according to the literature, between 40 and $70 \%$ (11-13). In the study group, arthritis was documented in 8 cases (40\%). Other musculoskeletal involvements were represented by inflammatory low back pain: 10 cases $(50 \%)$. Imaging test were performed and sacroiliitis was diagnosed in 4 cases $(20 \%)$.

\section{Severe organ involvement}

Ophthalmological involvement was present in 6 cases, posterior uveitis (5 cases), one patient presented chorioretinitis. One uncontrolled ophthalmologic involvement in the form of posterior uveitis led to vision loss.

Recurrent venous thrombosis was observed in 6 cases while 1 case presented thrombosis of the right atrium and inferior vena cava. Pulmonary vasculitis was seen in one case, in relation to a severe cardiovascular involvement. Neuro-Behçet's disease was diagnosed in 3 cases, 2 of those patients presented cerebral involvement, sustained by cerebral imaging, while isolated lesions of the spinal cord were seen in 1 case-transverse myelitis, also being the first sign of the disease.

\section{TREATMENT}

The type of clinical manifestations and organ involvement determined the course of treatment. 15 patients $(75 \%)$ received colchicine and in 9 patients (45\%) cortisone was added for bipolar aphthous lesions and for skin involvement.

TABEL 2. Treatment options in the study group ( $n=20$ patients)

\begin{tabular}{|l|c|}
\hline Treatment & Patients \\
\hline Colchicine & $15(75.0)$ \\
\hline Corticotherapy & $9(45.0)$ \\
\hline Immunosuppression & $10(50.0)$ \\
\hline CYC pulse therapy & $8(40.0)$ \\
\hline Azathioprine & $2(10.0)$ \\
\hline
\end{tabular}

Immunosuppression was necessary due to severe systemic involvement in 10 cases (50\%). Pulse therapy with cyclophosphamide was initiated in $8(40 \%)$ cases and the other $2(10 \%)$ received azathioprine. For those that received monthly pulse therapy with cyclophosphamide, remission was obtained in a 6 to
20 months and the cumulative dose of cyclophosphamide was between 3,6 grams and 20 grams. Remission was maintained using azathioprine in all cases. 5 patients $(25 \%)$ presented reactivation of the disease under treatment and another course of cyclophosphamide immunosuppressive therapy was needed.

\section{Correlations between disease activity and damage}

All patients presented active disease at the time of the diagnosis. Disease activity indexes: BVASv3 and BDCAF2006 and damage scores (VDI) were calculated. The mean scores for BVAS and BDCAF $\pm \mathrm{SD}$ at the time of the diagnosis were $7.95 \pm 4.36$ and $3.75 \pm 1.20$.

In the clinical case series, Spearman's rank correlation coefficient between BVAS v3 and BDCAF was strong $r=0.862$ and statistically significant $\mathrm{p}<$ 0.001 .

The outcome analysis after remission was calculated and rank correlation coefficient between VDI, and both BVASv3 and BDCAF was moderate (VDI-BVASv3 r=0.747, $\mathrm{p}<0.001$, VDI- BDCAF $\mathrm{r}=$ $0.795, \mathrm{p}<0.001)$.

As for immunosuppression induction decision and activity scores, the correlation coefficient was stronger for BVASv3 $\mathrm{r}=0.734$, and moderate for BDCAF2006 $r=0.647$ with $p<0.001$.

\section{Correlation between damage and immunosup- pressive therapy}

There was a moderate correlation between immunosuppressive treatment and and damage -VDI $(\mathrm{r}=0.700, \mathrm{p}<0.001)$.

The cause of damage (i.e. vasculitis vs. treatment) is not taken into consideration when VDI is calculated. We tried to observe if there are any connections between this and immunosuppression duration.

Data analysis showed the presence of mild correlation and no statistical impact between cyclophosphamide treatment duration and damage calculated as VDI $(r=0.474, p=0.36)$. This results show that long term treatment with immunosuppressant is not the cause for new damage items.

In contrast, when rank correlation coefficient between cortisone therapy and VDI was calculated, a moderate statistical impact was observed $(r=0.609$, $\mathrm{p}<0.001)$. 


\section{DISCUSSIONS}

Classification criteria are guidelines elaborated in order to help the physician in establishing a diagnosis. Not all classification criteria are met when the patient seeks medical help, signs and symptoms appear after a few months and others after years of disease. The main purpose is to establish early diagnosis and start treatment as soon as possible. It is very important to understand that signs and symptoms do not appear all at once in the beginning. Sometimes, it takes years until the patient presents all manifestations and diagnosis is obvious. During this time, irreversible damage may occur without treatment. In the study group, 10 patients out of 20 met the diagnosis criteria showing a sensibility of $50 \%$ compared to the $97 \%$ sensitivity according to studies. This finding is related to the small number of patient included in our study $(3,14,15)$.

Although pathergy is not a mandatory item in the diagnostic criteria, it is extensively tested. We have performed this test in all patients included in the study and in $13(65 \%)$ cases was positive. It is non-specific and is geographically related being more frequently on the "ancient silk road". The most recent studies show that only half of the patients in the middle and far east have positive pathergy (1517).

According to the latest information, the presence of HLA-B51gene is responsible for $32-52 \%$ of BD cases in different geographic subgroups. These values are substantially higher than a previous belief derived from a study on family association, which indicated that $19 \%$ of the genetic susceptibility to BD would be explained. Since it is not diagnostic, it is not performed usually. Data collected from the study group showed that genetic testing was performed in only 6 cases, and 5 were positive $(83,3 \%)$ $(18,19)$.

A systemic and careful assessment of disease's activity is necessary in order to decide the appropriate use of immunosuppressive therapies. Since vasculitis are chronic conditions with periods of activity and remission, organ damage is bound to appear. It is necessary to be able to differentiate between an acute lesion with reversible potential and damage, irreversible. There is no marker to reliably show disease activity in vasculitis. In the last years, a lot of clinical trials for different types of vasculitis were developed, and in order to diminish the inter observer variability and to transform the clinical evaluation of patients in a more objective process, multiple indexes were developed to calculate disease activity and damage. The existing medical literature shows that the regular use of disease activity and damage indexes in the clinical practice can offer a more structured approach to the patient with vasculitis. This can also help to justify treatment choices, especially when immunosuppression therapy is needed.

BVAS is one of the most used activity scores in clinical trials and even in clinical practice. Searching the literature for comparison of different activity indexes, a study performed in 2009 for the Vasculitis Clinical Research Consortium by PA Merkel, DD Cuthbertson and co Comparison of disease activity measures for anti-neutrophil cytoplasmic autoantibody (ANCA)-associated vasculitis, that tried to evaluate the ability of multiple disease activity indexes in granulomatosis with poliangiitis. The study concluded that all six activity indexes used for assessment of vasculitis patients were highly reliable and correlated (15,20-22).

For the group of patients diagnosed with Behçet's disease, the correlation between the two activity indexes was strong. Both indexes correlated approximately the same, with little difference with damage, long term cortisone therapy. The area in which the two indexes parted was the correlation with decision to start immunosuppressive therapy where BVAS v3 presented a stronger correlation. There are two variables that can influence this aspect, the most obvious is the small group of patients included in the study and the other one is that in BVAS v3 each organ involved is scored a different number of points in comparison with BDCAF2006 were any organ involvement records the same amount of points.

When vasculitis damage index is calculated, the cause of organ damage is not taken into account, weather it is an irreversible organ damage caused by vasculitis or it is an adverse reaction to treatment. This information is included in VDI definition. The data showed that damage progression is not influenced by prolonged immunosuppressive therapy, it is rather influenced by long term cortisone therapy use and disease activity(calculated as BVASv3 and BDCAF2006). This finding can be related with all the information presented in the literature and with treatment trends in the last years in which there is a new approach toward cortisone use in systemic diseases eg. Vasculitis. In spite of their powerful anti-inflammatory effect, the medical society becomes more and more aware of the adverse reactions of 
long term cortisone therapy (osteoporosis, increased risk for cardio thrombotic events, glaucoma, cataract etc.) and their input on the quality of life, for some years now. Since no treatment come's without risks, immunosuppressive agents like cyclophosphamide and azathioprine also present a large variety of adverse reactions (infections, karyokinesis). A systematic and through follow-up is usually able to detect early and prevent unfortunately events $(22,23)$.

\section{Strengths}

One of the few studies identified in the literature that tries to compare activity disease indexes and find correlations with damage and treatment decisions in a group of patients with Behçet's disease. The selected patients were admitted in a Department of Internal Medicine and Rheumatology, and were not part of a clinical trial, and were treated according to their physician's assessment, independently to the use of this activity indexes.

\section{Limitations of the study}

The study included a small number of cases $(n=20)$, from a non-endemic area for Behçet's disease. The internal medicine and rheumatology department is a reference center for autoimmune disease and vasculitis, so more complicated cases may have been admitted and the population might not be representative. This is a retrospective study that analyzed data collected from the information recorded in the hospital observation chart.

\section{REFERENCES}

1. International Team for the Revision of the International Criteria for Behçet's Disease (ITR-ICBD) et al. The International Criteria for Behçet's Disease (ICBD): A collaborative study of 27 countries on the sensitivity and specificity of the new criteria. J Eur Acad Dermatol Venereol. (2014) https://www.ncbi.nlm.nih.gov/ pubmed/23441863

2. International Study Group for Behçet's Disease. Criteria for diagnosis of Behçet's disease. Lancet. 1990 May 5

3. Davatchi F. Diagnosis/Classification Criteria for Behçet's Disease Pathology Research International Volume 2012 https://www. hindawi.com/journals/pri/2012/607921/

4. Mukhtyar C. et al. Modification and validation of the Birmingham Vasculitis Activity Score (version 3)

5. Nowatzky J. et al. Biomarkers in Behçet's Disease: Diagnosis and Disease Activity Int J Clin Rheumatol. 2009

6. de Souza Neves F. et al. Cross-cultural adaptation of simplified version (s) of Behçet's Disease Current Activity Form (BDCAF) and comparison between two different instruments with Brazilian versions for evaluating Behçet's Disease Activity: BR-BDCAF and BR-BDCAF(s) Rev Bras Reumatol, 2009

7. Flossmann O. et al. Development of comprehensive disease assessment in systemic vasculitis Ann Rheum Dis. 2007 Mar

\section{CONCLUSIONS}

Clinical manifestations of $\mathrm{BD}$ are polymorphic and the classification criteria are not always met at the time of the diagnosis. The most characteristic manifestation of the disease is the presence of aphtous lesions. Cardio thrombotic events, pulmonary and neurological involvement are the most serious manifestations and they require immunosuppressive therapy. Disease evolution and treatment choices are influenced by the systemic involvement.

Using disease activity indexes can be helpful in clinical practice. This way, an objective assessment of disease's activity can be obtained. There is a moderate to strong correlation between disease activity indexes, immunosuppressive treatment initiation and damage progression. This way, activity disease scores (BVASv3 and BDCAF2006) seem to be reliable tools to be used in anticipating the need for immunosuppression.

Comparing the two activity indexes, it resulted that BVASv3 correlates stronger with the need for immunosuppressive treatment than BDCAF2006. But both scores are equally able to anticipate damage.

Damage progression (VDI) was correlated stronger with long-term cortisone use, rather than immunosuppressive therapy. The damage index (VDI) is increased by irreversible organ damage due to disease activity and long term cortisone use, but not by the immunosuppressive therapy.

\section{Conflict of interest: none declared Financial support: none declared}

8. Coban Gul F. et al. Activity Criteria in Behçet's Disease https:// www.intechopen.com/books/authors/behcet-s-disease/activitycriteria-in-beh-et-s-disease

9. VDI Modified from Exley A.R., Bacon P.A., Luqmani et al. (1997) Development and initial validation of the VDI Arthritis Rheum

10. Luqmani R.A. Disease assessment in systemic vasculitis J Indian Rheumatol Assoc 2003

11. Fessler B.J. Vasculitis and related diseases, in Arthritis and Allied Conditions, W.J. Koopman and L.W. Moreland, Eds., pp. 18351844, Lippincott Williams and Wilkins, Philadelphia, Pa, USA, 2005.

12. Yazici H., Yurdakul S., Hamuryudan V., Fresko I. Behçet's syndrome, in Rheumatology, J. H. Klippel and P. A. Dieppe, Eds., pp. 1665-1670, Mosby, London, UK, 2003.

13. Yurdakul S., Yazıcı H., Tuzun Y. et al. The arthritis of Behçet's disease: prospective study, Annals of the Rheumatic Diseases, vol. 42, pp. 505-515, 1983. View at Google Scholar

14. Blake T. et al. Birmingham Behçet's service: classification of disease and application of the 2014 International Criteria for Behçet's Disease (ICBD) to a UK cohort BMC Musculoskeletal Disorders (2017)

15. Davatchi F. et al. How to deal with Behçet's disease in daily practice. Int J Rheum Dis. 2010 May;13(2):105-16. doi: 10.1111/j.1756-185X.2010.01462.x. 
16. Yazici $\mathrm{H}$. et al. A comparative study of the pathergy reaction among Turkish and British patients with Behçet's disease. Ann Rheum Dis. 1984

17. Seema Kalra, Alan Silman et al. Diagnosis and management of Neuro-Behçet's disease: international consensus recommendation J Neurol. 2014

18. Mathilde de Menthon et al. HLA-B51/B5 and the Risk of Behcet's Disease: A Systematic Review and Meta-Analysis of Case-Control Genetic Association Studies Arthritis Rheum. 2009

19. http://www.behcetdiseasesociety.org/menu/25/what-is-behcetsdisease
20. Merkel P.A. et al. Comparison of disease activity measures for antineutrophil cytoplasmic autoantibody (ANCA) - associated vasculitis Ann Rheum Dis. 2009 Jan

21. Merkel P. A. et al. The OMERACT Core Set of Outcome Measures for Use in Clinical Trials of ANCA-associated Vasculitis $J$ Rheumatol. 2011 July

22. Ponte $\mathbf{C}$. et al. Optimisation of vasculitis disease assessments in clinical trials, clinical care and long-term databases

23. Miller A. et al. An approach to the diagnosis and management of systemic vasculitis revised version with tracked changes removed Clin Exp Immunol. 2010 May 